\title{
Diagnosis of dengue- an overview
}

\author{
Rabindran ${ }^{1}$, Gedam D. $\mathrm{S}^{2}$
}

${ }^{1}$ Dr. Rabindran, Consultant, Neonatologist, Billroth Hospital, Chennai, India, ${ }^{2}$ Dr. D. Sharad Gedam, Professor of Paediatrics, R. K. D. F. Medical College \& Associated S. R. K. University, Bhopal, MP, India.

Address for Correspondence: Dr. Rabindran, E- mail: rabindranindia@yahoo.co.in

The first investigation ordered on clinical suspicion of dengue is complete blood count. The classical dengue triad includes increased hematocrit, atypical plasmacytoidlymphocytosis and thrombocytopenia [1]. Hemoconcentration (hematocrit elevated $>45 \%$ or by $>20 \%$ from previous value) is diagnostic of Dengue Hemorrhagic Fever. Further confirmation is usually made with Dengue Antigen test. During early stages of the disease around 4-5 days of fever onset, virus isolation, nucleic acid or antigen detection are used to diagnose the virus from serum, plasma or tissues. However serology remains the method of choice during convalescence. Other tests include antibody titre for dengue virus types, Polymerase chain reaction (PCR), Liver function tests, Serum protein \& albumin levels \& Coagulation panel.

Keywords: Dengue, MAC-ELISA, Biosensors.

Lab criteria for dengue diagnosis include any one of the following 4 tests-

1) Fourfold change in immunoglobulin G ( $\operatorname{IgG}$ ) or IgM antibody titre to 1 or more dengue virus antigens [2]

2) Tissue dengue virus antigen assay by immunohistochemistry/ immunofluorescence or serum enzyme immunoassay (MAC-ELISA, IgG ELISA, nonstructural protein 1 [NS1] ELISA, EIA)[1]; MAC-ELISA has $90 \%$ sensitivity \& $95 \%$ specificity when done 5 days after onset of fever. Different formats like capture ELISA, capture ultramicro ELISA, dot-ELISA, Au Bio DOT IgM capture \& dipsticks are available [3]. Dengue NS1 antigen can be detected as early as first day post onset of symptoms and remains detectable upto 18 days. Microsphere-based immunoassays (MIAs) based on covalent bonding of antigen or antibody to microspheres or beads \& detection using lasers to elicit fluorescence of varying wavelengths is faster than MAC-ELISA. Plaque Reduction and Neutralization Test (PRNT) \&microneutralization PRNT are the most specific serological tools for dengue antibody detection.

3) Viral genomic sequences in tissue, serum or cerebral spinal fluid (CSF) by reverse-transcriptase PCR (RT-PCR) assay [4]; RT-PCR has $80-90 \%$ sensitivity \& more that $95 \%$ specificity [3]. Real time RT-PCR based on Taq Man / SYBR green, NASBA (nucleic acid sequence based amplification) assay and RT-LAMP are recent advances in molecular techniques [5]. NASBA assay is an isothermal RNA-specific amplification assay which includes an initial reverse transcription, RNA amplification \& detection by electro-chemiluminescence or fluorescent-labelled molecular beacon probes [6].

4) Isolation of dengue virus from serumusing Cell culture. The mosquito cell line C6/36 (cloned from Ae. albopictus) or AP61 (from Ae. Pseudocutellaris) are the host cells of choice. Mammalian cell cultures like Vero, LLCMK2, and BHK21 are less efficient. Continuous lines of vertebrates, such as VERO, BHK-21, LLC-MK2, inoculation into animals and mosquitos are also used. The isolated viruses are detectedby indirect immunofluorescence using monoclonal antibodies against all four serotypes [7]. However it takes 1-2 weeks.

Characteristic lab findings also include mildmoderate elevation of aspartate aminotransferase and alanine aminotransferase values [8]. In severe dengue increased hematocrit, Hypoproteinemia, Prolonged prothrombin time \& activated partial thromboplastin time, Decreased fibrinogen \& Increased amount of fibrin split products are present. Guaiac testing for occult blood in stool \& hematuria may be present. Imaging studies like Chest radiography, Head computed tomography (CT) scanning to detect intracranial bleeding / cerebral edema due to severe dengue, 
Ultrasonography to detect ascites, pleural effusion, pericardial effusion and thickened gallbladder wall may be contributory [9]. Biosensors is newer technology which is rapid, sensitive, specific, qualitative \& quantitative. Types cDNA, IgM, IgG, Glycoprotein-E, NS1 protein and viral particles are used as different analyte probes. Different of biosensorsinclude Piezoelectric sensors, Optical biosensors \& Electrochemical biosensors. Electrochemical impedance spectroscopy monitors changes in capacitance associated with targets binding to prepared receptive electrode \& can be used for analysis of classical antigen- antibody binding events. A disposable paper based device known as Made-in-Singapore rapid test kit detects dengue-specific antibodies from saliva within 20 minutes $\&$ also differentiates between primary and secondary dengue infection [10]. Ferritin recently has been studied as a marker for differentiating dengue infection from other febrile illnesses of infective/ inflammatory etiology even in the absence of a positive NS1 antigen or a positive $\operatorname{IgM}$ antibody for dengue [11].

Khandelwal $\mathrm{R}$ et al in this issue observed that early febrile period of dengue fever, majority of patients have normal white blood cell count. Any change in the values of total leucocyte count points towards the progression of the disease severity [12].

Since dengue produces a broad spectrum of nonspecific symptoms clinical diagnosis is unreliable. As the disease worsens rapidly prompt early laboratory confirmation \& early intervention may be life-saving.

Funding: Nil, Conflict of interest: None initiated, Permission from IRB: Yes

\section{References}

1. Wiwanitkit V. Dengue fever: diagnosis and treatment. Expert Rev Anti Infect Ther. 2010 Jul;8 (7) : 841-5. doi: 10.1586/eri.10.53.

2. Kowitdamrong E, Thammaborvorn R, Semboonlor L, Mungmee V, Bhattarakosol P. Detection of dengue HI and $\operatorname{IgM}$ antibody: is it diagnostically useful? when and how? J Med Assoc Thai. 2001 Jun;84 Suppl1:S148-54.
3. VirojWiwanitkit. The Importance of Accurate Diagnosis of Dengue Fever; Future Virology. 2012;7(1):53-62.

4. Peh AE, Leo YS, Toh CS. Current and nanodiagnostic tools for dengue infection. Front Biosci (Schol Ed). 2011 Jun 1;3:806-21.

5. Dinesh Subedi, Andrew W. Taylor-Robinson. Laboratory Diagnosis of Dengue Infection: Current Techniques and Future Strategies. Open Journal of Clinical Diagnostics; Vol.4 No.1(2014), Article ID: 44045,8 pages. DOI:10.4236/ojcd.2014.41012.

6. Shu PY, Huang JH. Current advances in dengue diagnosis. ClinDiagn Lab Immunol. 2004 Jul;11 (4): $642-50$.

7. Henchal EA, Gentry MK, McCown JM, Brandt $\mathrm{W}$ E. Dengue virus-specific and flavivirus group determinants identified with monoclonal antibodies by indirect immunofluorescence. Am J Trop Med Hyg. 1982 Jul;31(4):830-6.

8. Simmons CP, Farrar JJ, Nguyen vV, Wills B. Dengue. N Engl J Med. 2012 Apr 12; 366 (15): 1423-32. doi: 10.1056/NEJMra1110265.

9. Ranjit S, Kissoon N. Dengue hemorrhagic fever and shock syndromes. Pediatr Crit Care Med. 2011 Jan; 12(1):90-100.doi:10.1097/PCC.0b013e3181e $911 \mathrm{a} 7$.

10. Zhang Yi, Bai J, Ying J. A stacking flow immunoassay for the detection of dengue-specific immunoglobulins in salivary fluid. Royal Society of Chemistry. Lab on a Chip. 2015 (6);doi: 10. 1039/c4lc 01127a.

11. C. A. M. van de Weg, R. M. H. G. Huits, C. S. Pannuti et al., Hyperferritinaemia in Dengue Virus Infected Patients Is Associated with Immune Activation and Coagulation Disturbances. PLoS Neglected Tropical Diseases, vol. 8, no. 10, 2014. doi.org / 10.1371/journal.pntd.0003214.

12. Khandelwal R, Khandelwal LM. Effect of dengue fever on the total leucocyte count and neutrophil count in children in early febrile period. Int J Pediatr Res. 2017; 4(10): 617-622. doi:10. 17511/ijpr. 2017.10.06.

\section{How to cite this article?}

Rabindran, Gedam D. S. Diagnosis of dengue- an overview. Int J Pediatr Res. 2017;4(10):588-589. doi: 10.17511/ijpr.2017.i10.01. 